\title{
AN ASSIST TOOL FOR PARALLEL PROGRAMMING
}

\author{
Kyoko Iwasawa \\ Computer Science Dept. Takushoku University \\ Hachioji Tokyo 193-0985, Japan
}

\begin{abstract}
We propose a tool to enable even beginners in parallel processing to develop a parallelization program using Open Multi-Processing (OpenMP) directives. Our proposed tool is characterized by its analysis of source programs for C and OpenMP directives written by users and its display of parallel structure diagrams. Further, the discovery of source program bugs is facilitated by the static analysis of interactive data access regions and decisions on the feasibility of parallelization using these parallel structure diagrams. While our proposed tool currently handles only basic OpenMP directives, our aim is to improve the analysis of parallel structure diagrams by including more complex simultaneous processing and more precise data access.
\end{abstract}

\section{KEYWORDS}

Parallel Execution, OpenMP Directive, Data Flow Analysis, Access Region, Parallel Structure Graph

\section{INTRODUCTION}

While the recent years have seen a proliferation in systems capable of parallel execution, including multicore and General purpose computing on graphics processing units (GPGPU), in general, the development of programs for parallel execution is difficult. While this is also the case with algorithm development, writing parallel processing code in an editing environment for the coding of sequential processing easily produces errors. Further, it is difficult to identify the errors, because in parallel programs the execution results are not reproducible.

Therefore we propose a programming environment particularly for beginners in parallel processing, using a parallel structure that is easily understood visually and also statically analyses the feasibility of parallel execution from the execution statement data access regions during program editing. We are developing the prototype of this tool.

Open Multi-Processing (OpenMP) is an application programming interface that supports multi-platform shared memory multiprocessing programming by the OpenMP Architecture Review Boards (OpenMP ARB). The details of OpenMP spec are written in (Chapman B., et al, 2008) and (Chandra R., et al, 2000). There are several tools for OpenMP programming. (Harnandez O., et al, 2004) and (Boosting parallel computing research) are integrated tools for OpenMP programming, which include compiler and parallel execution environments. They have various functions and can be somewhat difficult for beginner of parallel programming. We simplify the analyzing method on (Iwasawa K., 1998) and (Watanabe T., et al, 2007) because our proposed tool does not generate parallel object code, but suggests user appropriate directives for parallelization.

\section{PROPOSED TOOL OVERVIEW}

Our proposed tool is an environment for the $\mathrm{C}$ programming language used in creating and editing programs that give parallelization directions using OpenMP directives. It has the following three main functions.

(1) OpenMP directive analysis

(2) Parallel structure graph display

(3) Interactive, static data flow analysis, and parallelization feasibility decisions.

(4) Profiling behaver of parallel execution and its display 
In addition, it displays the structure written in OpenMP in an easy understood manner for users not accustomed to parallel processing, as well as for beginners to perform debugging by displaying static analytical results interactively. Figure 1 shows the overall proposed tool structure.

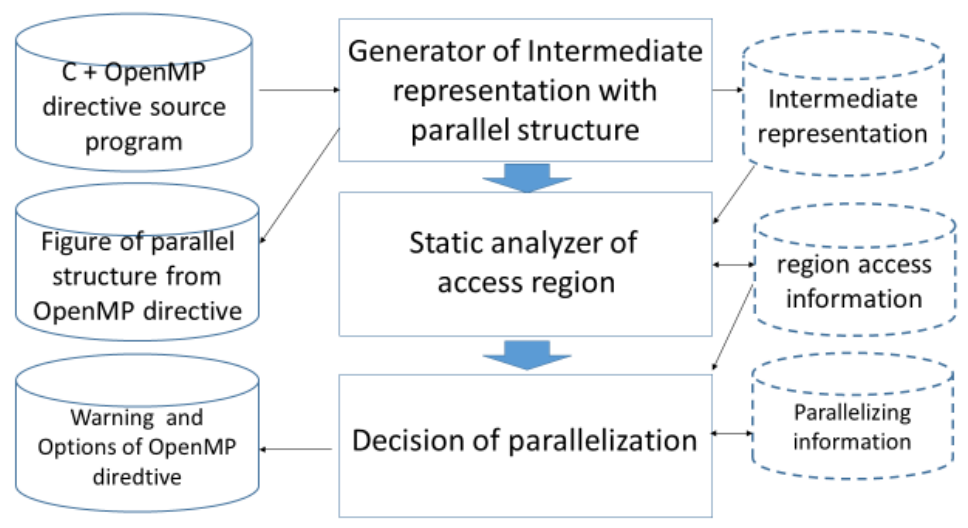

Figure 1. Overall tool structure

OpenMP directives within $\mathrm{C}$ source programs are analyzed and transformed to intermediate representation. Subsequently this is formed and displayed as the parallel structure diagram in Figure 2. In this diagram, the user selects the quadrangle in the execution direction and the elliptical shape in the parallel execution direction to decide the data access region and parallel execution feasibility.

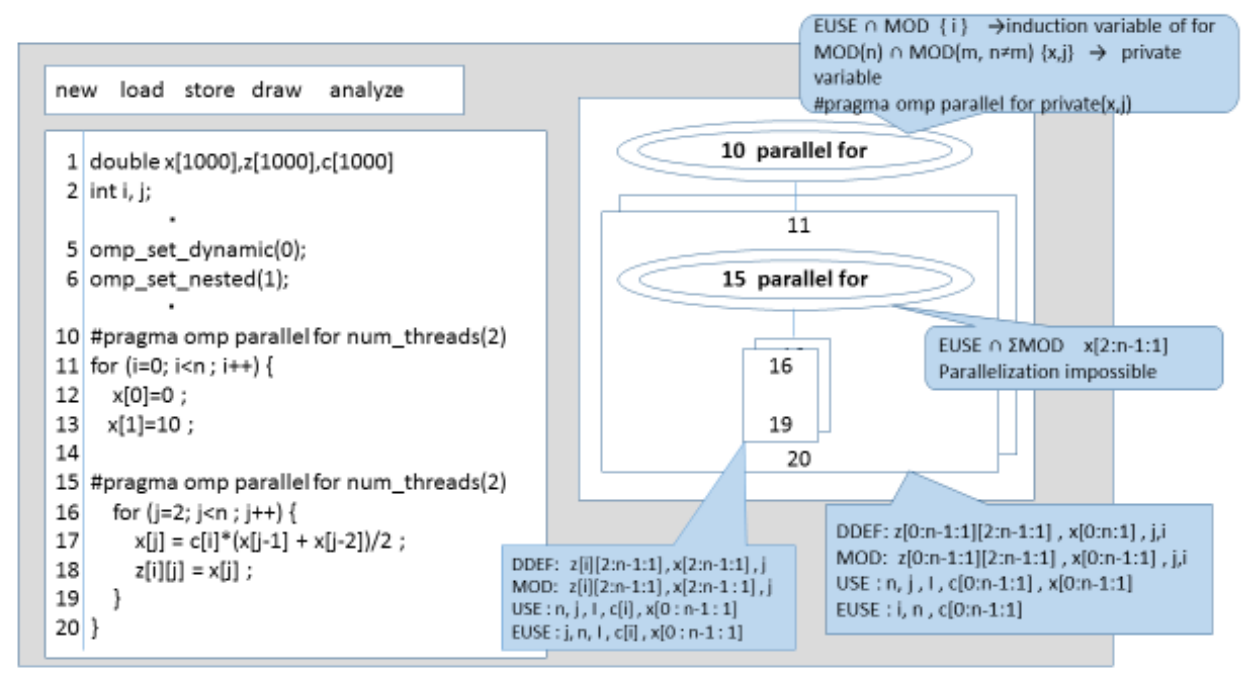

Figure 2. Display screen

Figure 2 shows the display screen and an analytical example. In the editing window on the left, the user performs the parallelization program coding using the $\mathrm{C}$ programming language script and OpenMP directives. The command, 'draw' is selected for the tool to display a parallel structure diagram on the left. The details of this parallel structure diagram are presented in Section III. The command 'analyze' is selected to enable the selection of the diagram quadrangle and elliptical shape (line number and OpenMP directive). Selecting one of these displays the parallel block access regions for that OpenMP directive and the parallelization decision. The balloons indicating the quadrangles in Figure 2 contain an example of the analysis results. 


\section{PALALLEL GRAPH DRAWING OF OPENMP DIRECTIVE ANALYTICAL RESULTS}

The tool analyzes syntax and context of OpenMP directives in the $\mathrm{C}$ source program, and these directives are reflected in the intermediate representation. This displays the diagram expressing the parallel structure in a graph from this intermediate representation. This is a graph structure with quadrangles expressing the parallel execution unit and ellipses expressing parallel execution direction a s nodes.

Quadrangles do not display the execution directions merely by inserting the first and last direction numbers. Ellipses have line numbers and OpenMP directives as labels.

Although there are many OpenMP directives, the current parallel structure diagrams are expressing only for the following three basic types thought necessary for beginners as subjects of analysis.

(1)\#pragma omp parallel

(2)\#pragma omp parallel for

(3)\#pragma omp parallel sections and \#pragma omp section

The "parallel for" for the do-all-type parallel processing is expressed in double ellipses, and their loops are expressed by overlapping quadrangles. The "parallel sections" that expresses parallel-case type parallel processing is single ellipses. The nested parallel execution are expressed by drawing ellipses and quadrangles in other quadrangles.

The requirements of parallel structure graph to express directives are following:

- To distinguish between the execution of same statements in parallel for the number of threads (\#pragma omp parallel) and the execution of different statements in parallel ( \#pragma omp parallel for, \#pragma omp parallel sections).

- To arrange statements to be executed simultaneously, side by side.

- To arrange sequential statements vertically and clarify the order of execution by using connected line.

- To show the synchronization point.

- To disclose parallel nesting structure.

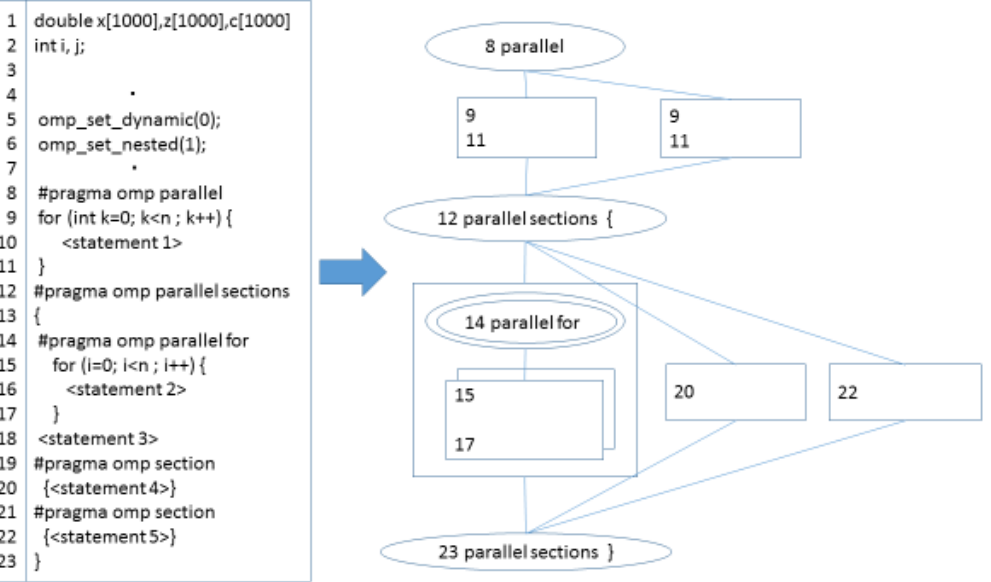

Figure 3. Source program and parallel structure diagrams

Figure 3 shows an example of source program and parallel structure graph. Since the eighth line is a "parallel" directive, it directs to execute the entire following for-loop parallel by the thread number. The other hand, the 14th line is a "parallel for" directive that divides the loop repetition and directs to execute divided them in a parallel manner. This is expressed with a double ellipse directive and overlapping quadrangles such that the difference can be intuitively understood. The 12th line is a "parallel section" directive, and the quadrangles are able to execute parallel and the synchronizing point for the "\}" in the 23rd line to clarify its scope. While internal statements of each quadrangle and the overlapping quadrangle execute sequentially, when there is any parallel directive graph shows nested parallel execution. 


\section{STATIC ANALYSIS OF DATA ACCESS REGION}

When user selects the quadrangle in a generated parallel structure graph from an OpenMP directive analysis, the tool finds and displays the data access region by its execution. Additionally, when user selects the ellipse that expresses parallel directive, the tools decides the parallel execution feasibility. An example is shown in Figure 2.

Access region analysis to decide parallelization feasibility analyses what regions are accessed in what order according to a control flow.

\subsection{Access Region}

Four data access types are available:

- $\quad$ Possible use (USE)

Data that might be used within a certain scope (flow graph pass)

- $\quad$ Possible exposed use (EUSE: Exposed USE)

Data that might be used within a certain scope before definition (flow graph pass)

- Possible definitions (MOD: MODified)

Data that might be updated within a certain scope before definition (flow graph pass)

- Definitely defined (DDEF: Definitely Defined)

Data that is definitely updated within a certain scope (flow graph pass)

The 'flow graph pass' above widens the scope of analysis to the parallelization block through the process of one statement $\rightarrow$ basic block $\rightarrow$ loop i-th iteration $\rightarrow$ all repetitions loop $\rightarrow$ outer loop.

While the 'possible use' and 'possible definitions' are control flow insensitive, 'possible exposed use' and 'definitely defined' are control flow sensitive. These regions are related as follows:

Possible use $\subseteq$ Possible exposed use

Possible definitions $\subseteq$ Definitely defined

As understood from the analytical methods in Section $\mathrm{V}$, the 'possible use' and 'possible definitions' are required to guarantee safety.

\subsection{Integration Methods of Data Access Regions}

In the if-then-else structure, when node 1 is 'then' and node 2 is 'else', the tool integrates the access regions as in Figure 4 (+ is union and $*$ is intersection).

After fusing the if-then-else structure, the nodes sometimes line up in a row. Node 1 is the priority node and node 2 is the next node. The tool integrates the access region as in Figure 5 (- is the difference set excluding the intersection set from the first operand).

Concerning loops, the access region of the i-th iteration is analysed by method 2 and method 3 and the access region of the entire loop is analysed as following Figure 6. The information of data access in a loop is expanded.

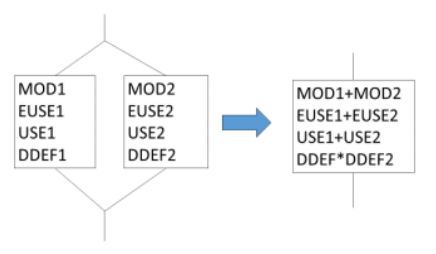

(a) fuse

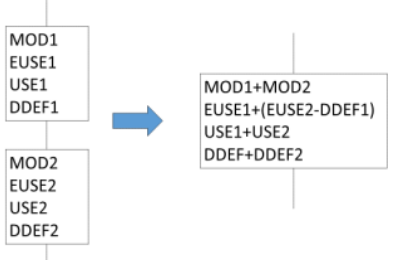

(b) join

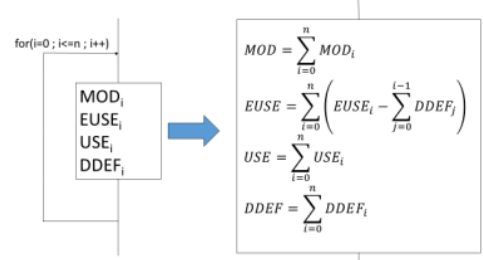

(c) expand

Figure 4. Access region integration 


\subsection{Parallel Execution Feasibility Decision}

By using the parallel structure graph, user knows feasibility of parallel execution. The tool decides whether each iteration of loop can be executed independently for the do-all type, and for the parallel-case type the tool decides whether the parallel blocks surrounded by the section directives can be executed independently. When dependency that impedes parallel execution occurs, tool displays it as the reason of impossible of parallelization. System decides if there is any critical dependency by calculating of the intersection of access regions.

Decisions of parallel execution are conducted using access regions as follows:

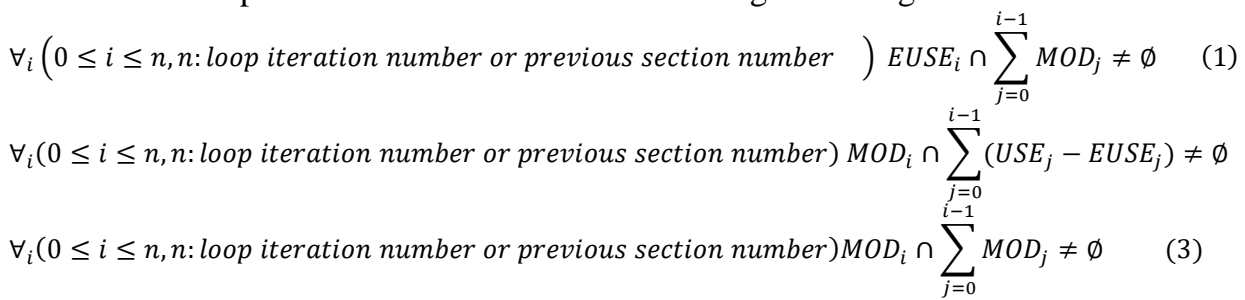

When condition (1) is satisfied, there is any data access that causes flow data dependence, and it becomes parallelization impeding factor. When conditions (2) and (3) are satisfied, there is any data access causes anti and output data dependence. In this case, parallelization might be possible by privatisation of these confirming data. The tool recommends users to add private clause to parallel for directive.

The balloon indicating the ellipse with the parallelization direction in Figure 2 contains an example of the analysis results.

\section{CONCLUSION}

In a structure as presented herein, providing a parallel program development environment allows the meaning of the written OpenMP directives to be easily understood and mistakes in directives to be easily recognized by beginners not accustomed to parallel processing. Further, this enables the detection and correction of errors peculiar to parallel processing at an early development stage for an accurate static analysis. Inserting OpenMP directives into $\mathrm{C}$ programs, such as parallel structures graph enables the easy understanding of parallel structure mistakes and missing synchronous processes because when necessary OpenMP directive is missed out the graph does not have parallel structure. Then the tool makes comments reason why the tool cannot make parallel structure.

Currently, the prototype of the proposed tool is under developing. The GUI specifications have developed as they are considered. We are going to connect the result of static analysis to parallel structure graph. In the future, we would like to increase the types of OpenMP directives for analysis, display complex synchronous processes in an easily understood manner, and provide appropriate advice from the analytical results.

On the other hand side, we are adding the dynamic analysis feature by profiling the parallel program. It might be useful to execute parallel programs efficiently.

\section{REFERENCES}

Boosting parallel computing research, Programming Models@BSC, https://pm.bsc.es/ompss.

Chandra R., et al, 2000. Parallel Programming in OpenMP Morgan Kaufmann Publishers, USA.

Chapman B., et al, 2008. Using OpenMP: Portable Shared Memory Parallel Programming, MIT Press, USA.

COINS-project, COINS Compiler Infrastructure, http://coins-compiler.osdn.jp/international/index.html.

Harnandez O., et al, 2004. Dragon: A Static and Dynamic Tool for OpenMP, International Workshop on OpenMP Applocation and Tools, Huston, USA, pp.54-66.

Iwasawa K., 1998. Automatic Parallelizing Method of Loops by Conditional Region Analysis. Proceedings of the 16th IASTED International Conference Applied Informatics, Innsbruck, Austria, pp.310-313.

The OpenMP ARB (Architecture Review Boards), HOME OpenMP, https://www.openmp.org.

Watanabe T. et al, 2007. Design assists for embedded systems in the COINS Compiler Infrastructure, Proceedings of the 10th International Workshop on Innovative Architecture for Future Generation High-Performance Processors and Systems, Hawaii, USA. pp.60-69. 